\title{
Efficient sound radiation using a bandgap structure
}

Jung, Jaesoon; Jeong, Cheol Ho; Jensen, Jakob S.

\section{Published in:}

Applied Physics Letters

Link to article, DOI:

$10.1063 / 1.5110296$

Publication date:

2019

Document Version

Publisher's PDF, also known as Version of record

Link back to DTU Orbit

Citation (APA):

Jung, J., Jeong, C. H., \& Jensen, J. S. (2019). Efficient sound radiation using a bandgap structure. Applied Physics Letters, 115(4), [041903]. https://doi.org/10.1063/1.5110296

\section{General rights}

Copyright and moral rights for the publications made accessible in the public portal are retained by the authors and/or other copyright owners and it is a condition of accessing publications that users recognise and abide by the legal requirements associated with these rights.

- Users may download and print one copy of any publication from the public portal for the purpose of private study or research.

- You may not further distribute the material or use it for any profit-making activity or commercial gain

- You may freely distribute the URL identifying the publication in the public portal

If you believe that this document breaches copyright please contact us providing details, and we will remove access to the work immediately and investigate your claim. 


\section{Efficient sound radiation using a bandgap structure}

Cite as: Appl. Phys. Lett. 115, 041903 (2019); https://doi.org/10.1063/1.5110296

Submitted: 15 May 2019 . Accepted: 10 July 2019 . Published Online: 24 July 2019

Jaesoon Jung, Cheol-Ho Jeong, and Jakob S. Jensen
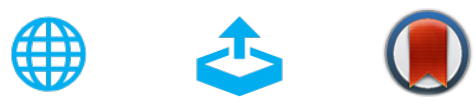

View Online

Export Citation

CrossMark

\section{ARTICLES YOU MAY BE INTERESTED IN}

One-dimensional surface phononic crystal ring resonator and its application in gas sensing Applied Physics Letters 115, 041902 (2019); https://doi.org/10.1063/1.5090592

Terahertz field depolarization and absorption within composite media Applied Physics Letters 115, 041901 (2019); https://doi.org/10.1063/1.5101068

In-phase supermode operation in GaN-based vertical-cavity surface-emitting laser Applied Physics Letters 115, 041101 (2019); https://doi.org/10.1063/1.5104289

\section{Applied Physics Letters}

Mid-IR and $\mathrm{THz}$ frequency combs special collection 


\title{
Efficient sound radiation using a bandgap structure
}

\author{
Cite as: Appl. Phys. Lett. 115, 041903 (2019); doi: 10.1063/1.5110296 \\ Submitted: 15 May 2019 • Accepted: 10 July 2019 . \\ Published Online: 24 July 2019

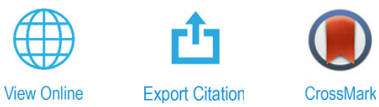

Jaesoon Jung, ${ }^{1, \text { a) }}$ Cheol-Ho Jeong, ${ }^{1}$ and Jakob S. Jensen ${ }^{2}$

AFFILIATIONS

'Department of Electrical Engineering, Technical University of Denmark, 2800 Kongens Lyngby, Denmark

${ }^{2}$ Department of Mechanical Engineering, Technical University of Denmark, 2800 Kongens Lyngby, Denmark

a)jjung@elektro.dtu.dk

\begin{abstract}
This work reports on the use of bandgaps to increase the efficiency of sound radiation employing defect modes on a phononic crystal (PnC). A PnC consisting of a 2D array of studs on an aluminum plate is considered, and a defect is created by removing four studs. Numerical simulations predict $8 \mathrm{~dB}$ higher radiation efficiency and significantly more uniform directivity of sound due to the piston-like defect modes that suppress interference between acoustic waves. An experimental study of the vibrational response is carried out in order to validate the numerical result. Comparisons of the radiation efficiency and the directivity index between the numerical and experimental results show good agreement. These findings may pave the way to use bandgap structures as effective acoustic radiators.
\end{abstract}

Published under license by AIP Publishing. https://doi.org/10.1063/1.5110296

For the last two decades, acoustic and elastic phononic crystals (PnCs) and metamaterials have received considerable attention due to their unique wave manipulation capabilities. ${ }^{1}$ The most well-known property is the ability to create phononic bandgaps that forbid the propagation of waves. ${ }^{2-5}$ Two common uses of bandgaps are attenuation of the transmission of the wave energy and guiding the propagation of waves. Based on these two elements, many interesting devices such as passive filters, ${ }^{6-10}$ wave guides, ${ }^{11-15}$ and acoustic lenses ${ }^{16-19}$ have been developed.

In addition to single wave applications, the use of bandgaps has been extended to interaction problems between elastic and acoustic waves, i.e., vibro-acoustics. ${ }^{20-27}$ The main focus of the vibro-acoustic applications of bandgap structures is the reduction of noise radiating from vibrating structures due to attenuation of vibrational energy. Liu et al. ${ }^{20}$ proposed a locally resonant sonic material that has a bandgap in the low frequency regime and blocks the sound transmission surpassing the mass density law. After this pioneering work, the noise insulation became one of the main bandgap applications.

It is generally accepted that the reduction of vibration results in the reduction of sound radiation or noise. However, in a recent study it was found that the efficiency of sound radiation increases and therefore may counterbalance the benefit of a bandgap for the noise reduction purpose. ${ }^{28}$ However, no attention has been given to a detailed treatment of the increased efficiency of sound radiation as well as the associated improved sound radiation directivity.
The increased efficiency of sound radiation could broaden the possibility to use bandgap structures as acoustic radiators. The key idea of our approach is to create a defect in a PnC that confines the vibrational energy. The confined vibrational energy creates piston-like defect modes that radiate sound more efficiently due to a reduced interference between acoustic waves. Such defect modes have been widely used to create photonic crystal laser devices. ${ }^{29,30}$ Recently, this principle was transferred to acoustics for harvesting acoustic energies by confining vibrations. ${ }^{27}$ Our work is in line with Ref. 27 , but we focus on the sound radiation. Figure 1 illustrates this idea by visualizing acoustic intensities radiated from the bare structure and the $\mathrm{PnC}$ with a defect. For this illustration, a finite element (FE) model using the structure-acoustic interaction module in COMSOL Multiphysics is used.

From Fig. 1, two clear benefits of confining the vibration with the $\mathrm{PnC}$ can be seen. First, the acoustic waves are not subjected to cancelation and second, the acoustic waves radiate more uniformly in the space. In this paper, we will demonstrate these benefits from numerical simulations and an experimental study.

In order to realize the idea, we consider the PnC consisting of a thin aluminum plate with periodically arranged rigid studs as shown in Fig. 2(a). The material properties of the aluminum are an elastic modulus of $68 \mathrm{GPa}$, Poisson's ratio of 0.33 , a mass density of $2700 \mathrm{~kg} / \mathrm{m}^{3}$, and a loss factor of 0.002 . These material properties are tuned to match the experiment results for the bare aluminum plate. To create a broad phononic bandgap in the audible frequency range, the mass 


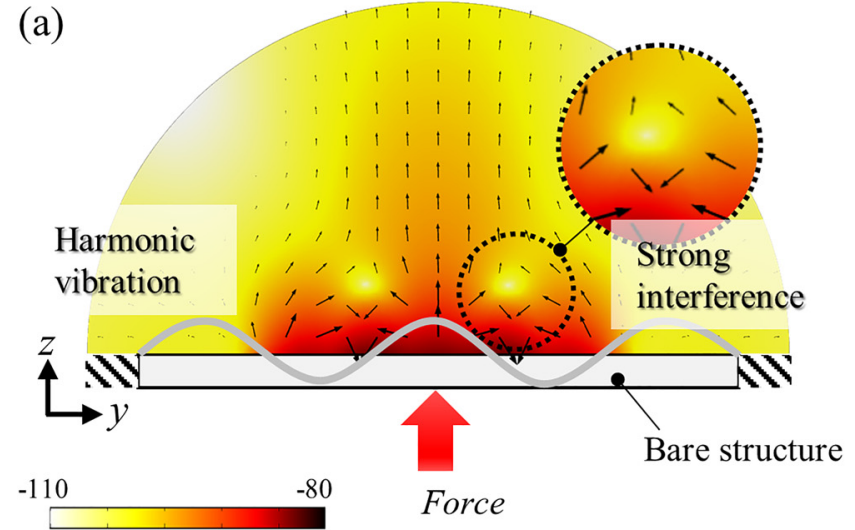

Intensity magnitude $[\mathrm{dB}]$

(b)

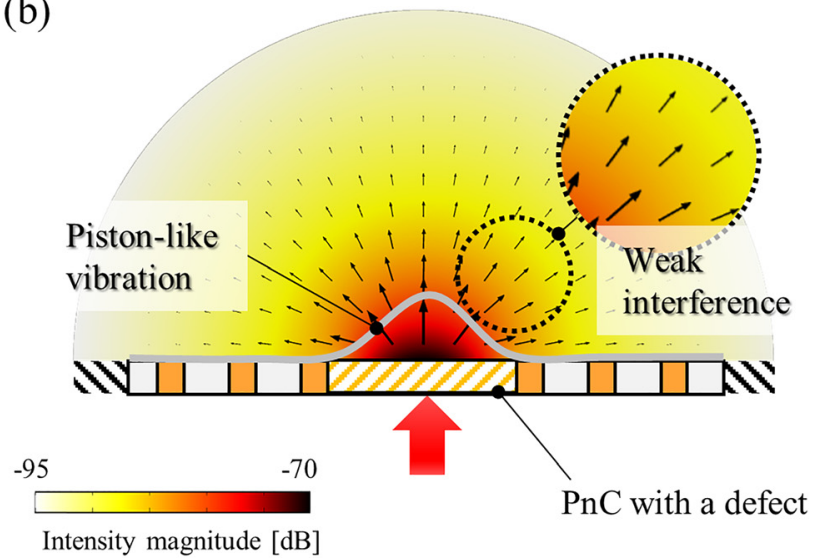

FIG. 1. Acoustic intensities radiating from the bare structure (a) and the $\mathrm{PnC}$ with a defect (b); the length of arrows represents the magnitude of acoustic intensities. The acoustic intensity is computed using the FE model described in Fig. 2(a) at $1850 \mathrm{~Hz}$.

of each stud is chosen to be $8.7 \mathrm{~g}$, resulting in a bandgap ranging from $1.9 \mathrm{kHz}$ to $9.6 \mathrm{kHz}$, as highlighted in Fig. 2(b).

The studs are assumed as rigid to simplify the simulation with no resonances induced by studs. For simulations concerning the resonances of studs, one can consider the stiffness of the studs with specific parameters. The hexagonal shape is chosen for the stud to match the shape with the practical fabrication, but a circular ${ }^{27}$ or rectangular ${ }^{11}$ shape can be considered with very similar results. The defect is created by removing four studs in the center part.

For the FE simulation, shell elements and solid elements are used for the aluminum plate and the studs, respectively. First, the vibrational velocity field of the $\mathrm{PnC}$ is computed with the harmonic body force $\left(1 \mathrm{~N} / \mathrm{m}^{3}\right)$ applied at the center of the $\mathrm{PnC}$ in the $\mathrm{z}$-direction. All boundaries of the aluminum plate are fixed. The sound pressure $p$ at a field point, which is $1 \mathrm{~m}$ away from the center of the surface, is calculated using the Rayleigh integral ${ }^{31}$ represented as

$$
p=\frac{j \omega \rho}{2 \pi} \int_{S} \frac{v e^{-j k R}}{R\left(\mathbf{r}, \mathbf{r}_{0}\right)} d S,
$$
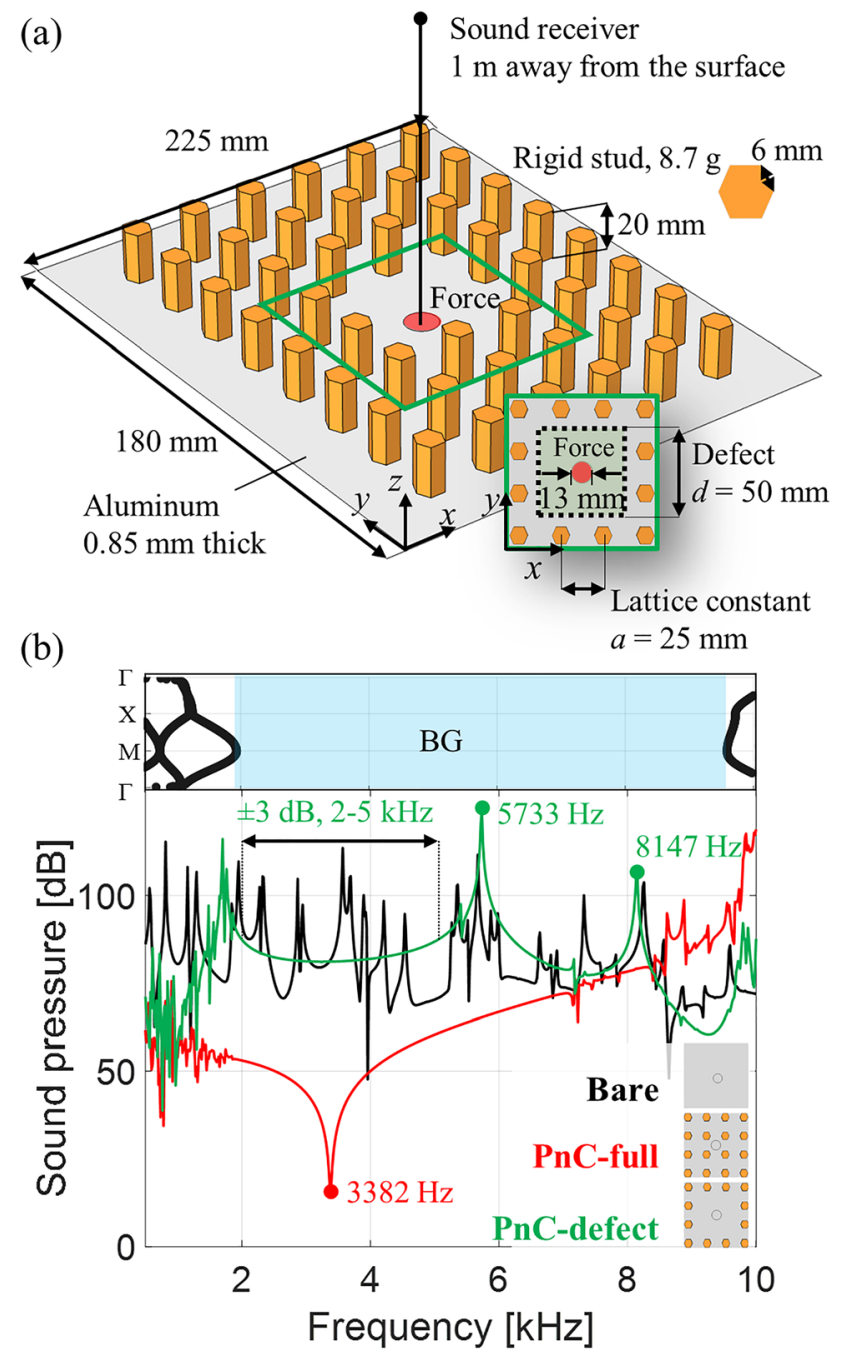

FIG. 2. (a) PnC with the defect, and (b) the sound pressure response calculated at $1 \mathrm{~m}$ away from the center of the surface. The reference sound pressure is $20 \mu \mathrm{Pa}$.

where $\omega$ is the angular frequency, $\rho_{0}$ is the mass density of air $\left(=1.2 \mathrm{~kg} / \mathrm{m}^{3}\right), v$ is the surface normal velocity on the plate, $k$ is the acoustic wave number $\left(=\omega / c_{0}\right)$ calculated from the speed of sound $c_{0}$ $(=343 \mathrm{~m} / \mathrm{s})$, and $R\left(\mathbf{r}, \mathbf{r}_{0}\right)$ is the distance between the surface point $\mathbf{r}_{0}$ and the observation point $\mathbf{r}$. The symbol $S$ represents the area of the surface. It should be emphasized that a baffled flat surface is assumed in this formulation. This is a reasonable assumption if we use the flat side of the PnC for sound radiation. The air loading effect is negligible since the acoustic waves are radiated to the open space ${ }^{31}$ and due to the use of a relatively stiff and dense material (aluminum).

Figure 2(b) shows the calculated sound pressure for the bare plate, the full $\mathrm{PnC}$, and the defect $\mathrm{PnC}$. The bare plate displays a highly fluctuating sound pressure in the frequency range of interest. Conversely, the full $\mathrm{PnC}$ shows a significantly reduced sound pressure within most of the bandgap. The dip occurring at $3382 \mathrm{~Hz}$ corresponds to an antiresonance of the vibration response. After the dip, 
the sound pressure increases gradually as the frequency increases. This sound deadening effect of the $\mathrm{PnC}$ is well known for noise reduction applications. $^{20-25}$ In contrast to this expectable behavior, the defect PnC displays a sound pressure that increases significantly within the bandgap. The averaged sound pressure of the defect $\mathrm{PnC}$ is $18 \mathrm{~dB}$ higher than the full $\mathrm{PnC}$ and $2 \mathrm{~dB}$ higher than the bare plate. In Fig. 2(b), it can be seen that the sound pressure of the defect PnC is comparable to the bare plate although the stiffness and the mass are increased significantly. However, the number of resonances is much smaller than that of the bare plate. Two clear resonances occur at $5733 \mathrm{~Hz}$ and $8147 \mathrm{~Hz}$. Due to the small number of resonances, a smooth sound pressure with only $3 \mathrm{~dB}$ deviation is obtained in a wide frequency range $(2-5 \mathrm{kHz})$. This result demonstrates that by creating the defect, the PnC can amplify and smoothen the sound pressure characteristics, which make it attractive as an acoustic radiator.

In order to further analyze the interesting behavior of the defect $\mathrm{PnC}$ for sound radiation, we employ the concept of radiation efficiency. Radiation efficiency $\sigma$ is a useful dimensionless quantity that quantifies how efficiently the structure radiates sound in comparison with the ideal piston. ${ }^{31-33}$ Generally, the radiation efficiency is defined as the ratio of sound power $\Pi$ between the structure and the baffled piston as

$$
\begin{gathered}
\Pi=\int_{S} \operatorname{Re}\left\{\frac{p v^{*}}{2}\right\} d S, \\
\sigma=\frac{\Pi}{\rho_{0} c_{0} \int_{S} v v^{*} d S},
\end{gathered}
$$

where the asterisk denotes the complex conjugate. To evaluate Eq. (3), the discrete calculation method described in Ref. 33 is used. Figure 3(a) displays the radiation efficiency for three structures, i.e., the bare plate, full $\mathrm{PnC}$, and defect $\mathrm{PnC}$. Averaged in the bandgap, the defect PnC shows $8 \mathrm{~dB}$ and $5 \mathrm{~dB}$ higher radiation efficiency than the bare plate and the full $\mathrm{PnC}$, respectively. The piston-like vibration pattern of the defect $\mathrm{PnC}$, which resembles the motion of a baffled piston as described in Figs. 3(b) and 3(c), is attributed to the high radiation efficiency. On the other hand, the distributed vibration of the bare plate described in Fig. 3(e) produces a poor and fluctuating radiation efficiency in the entire frequency range. The cancelation of acoustic wave contributions from adjacent areas are the main cause of this behavior as illustrated in Fig. 1(a). The full PnC also has piston-like (a)

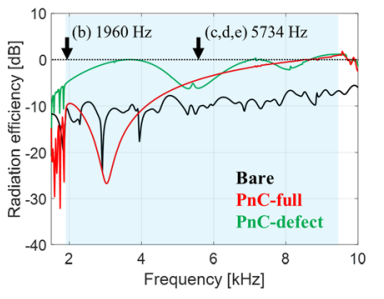

(b)

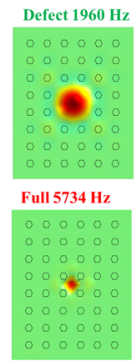

(c) Defect $5734 \mathrm{~Hz}$

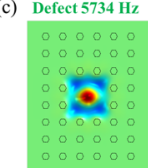

(e) Bare $5734 \mathrm{~Hz}$

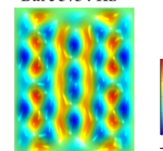

FIG. 3. (a) The radiation efficiency for the bare plate, the full $\mathrm{PnC}$, and the defect $\mathrm{PnC}$; the bandgap is highlighted. (b)-(e) The normalized vibrational velocity fields of structures at frequencies that represent the defect modes. vibrations confined to the small region as shown in Fig. 3(d). The small area of the confined vibration produces poor radiation efficiency at frequencies below $4 \mathrm{kHz}$, but it gradually increases as the frequency increases. It is worth noting that the full $\mathrm{PnC}$ displays a very smooth behavior in contrast to the slightly oscillating behavior of the defect PnC. From the results, we can conclude that a $\mathrm{PnC}$ with and without a defect can increase the efficiency of sound radiation significantly. It is worth noting that it should be possible to manipulate the piston-like vibrational behavior further by systematically designing the defect as described in Ref. 34 for a photonic crystal waveguide.

To validate the increased radiation efficiency obtained from the numerical result, a vibration experiment is carried out. For the experiment, the defect PnC is fabricated as shown in Fig. 4(a). The dimensions are the same as the FE simulation, and the rigid studs are made of fastened bolts and nuts. To adjust the mass of the studs to $8.7 \mathrm{~g}$, four nuts are stacked together. An electrodynamic exciter (Dayton Audio Daex 13ct) is attached at the center of the aluminum plate. The vibrational velocity distribution on the flat side is measured using the laser Doppler vibrometer (Polytec PSV-400) using a random signal

(a)
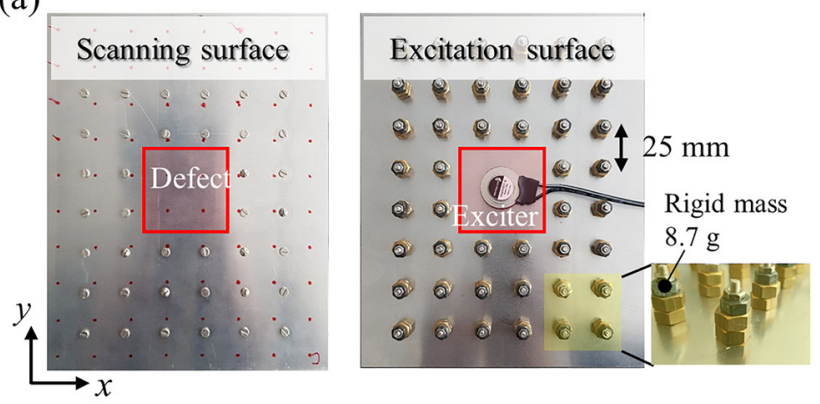

(b)
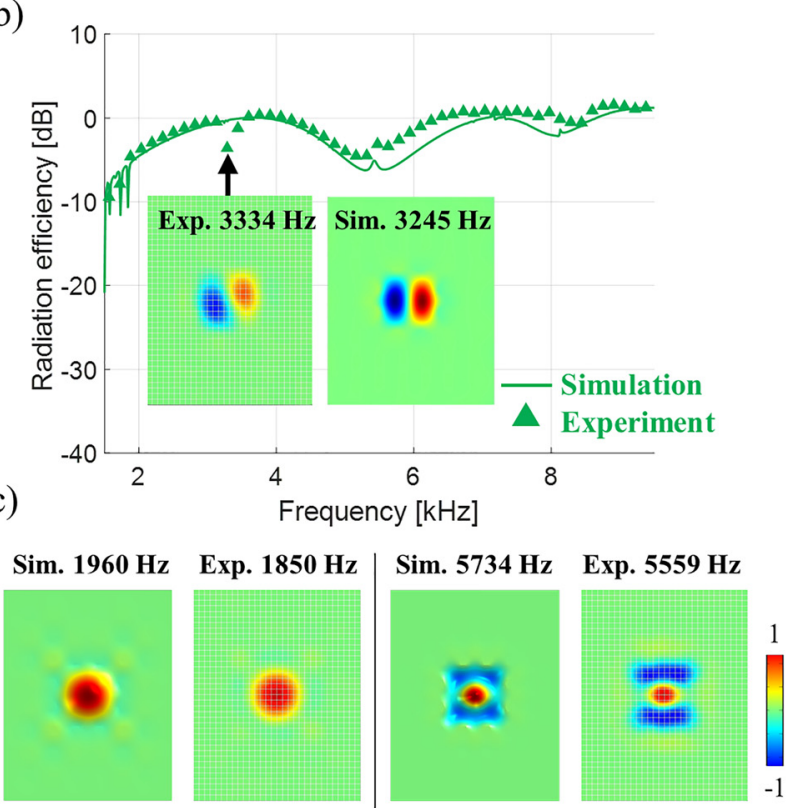

FIG. 4. (a) The fabricated PnC with defect, (b) the radiation efficiency calculated from the simulation and measurement results, and (c) the piston-like defect modes. 
excitation. The scanning resolution is $31 \times 39$ (i.e., 1209 points), which is dense enough to capture the bending wavelength of $4 \mathrm{~mm}$ at $10 \mathrm{kHz}$. A free boundary condition is applied for the setup, but within the bandgap frequency range the vibration levels are very low near the boundary, so the results are not sensitive to the boundary conditions. With the measured vibration velocity, the radiation efficiency is calculated using Eq. (3). Thus, our experimental validation is limited to the vibrational part and we rely on the use of the Rayleigh integral to quantify the corresponding acoustic field. In Fig. 4(b), we see that the two results are in good agreement except for the dip at $3334 \mathrm{~Hz}$. The dip occurs due to the dipole-like mode which is invisible in the simulation since the force is applied at the node of the mode shape. However, piston-like defect modes demonstrate a good correlation between the simulation and the measurement as shown in Fig. 4(c). In this figure, two defect modes are in good agreement in terms of shapes and frequencies.

One additional benefit of the presented approach is that the sound is more uniformly radiated in the space, a feature not achieved with the bare plate. To quantify the uniformity of sound radiation, the directivity index (DI), which is a dimensionless quantity measuring the uniformity of the sound field is calculated using the following formulation,

$$
D I(\omega)=10 \log _{10} \frac{|p(0,0, \omega)|^{2}}{\frac{1}{2 \pi} \int_{\phi=0}^{2 \pi} \int_{\theta=0}^{\pi / 2}|p(\theta, \phi, \omega)|^{2} \sin \theta d \theta d \phi},
$$

where $p(0,0, \omega)$ is the acoustic pressure at the point $1 \mathrm{~m}$ away from the center of the surface as described in Fig. 5. $p(\theta, \phi, \omega)$ is the acoustic pressure at the point away from the center by the elevation angle $\theta$ and the azimuth angle $\phi$. For the calculation of the DI, a hemispherical domain in front of the surface, ${ }^{35}$ where the elevation angle ranges from 0 to $\pi / 2$, is considered. If the sound is uniformly radiated, the DI

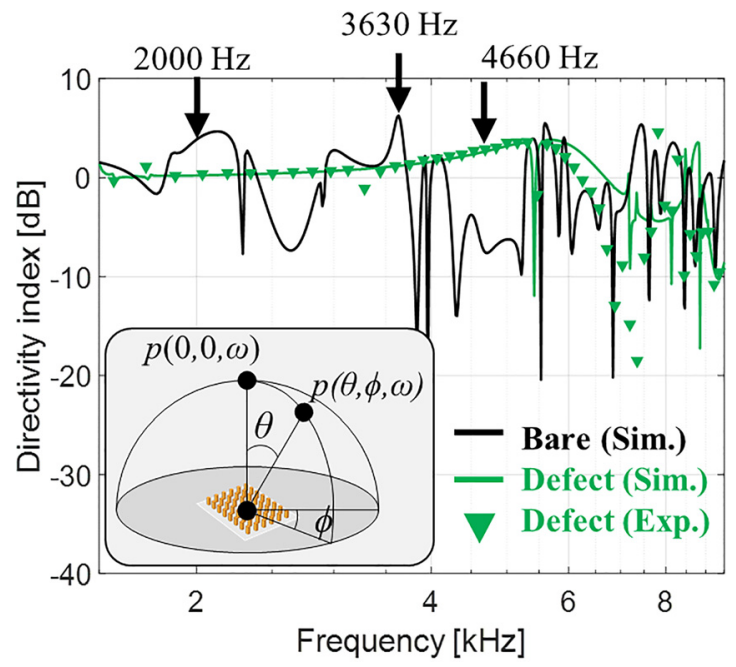

FIG. 5. The Dls for the bare plate, the PnC with the defect, and the description of the coordinate system for the DI calculation. is equal to zero and a nonzero value represents nonuniformity of the sound.

Figure 5 shows the DI for the bare plate and the defect PnC. For validation purposes, the DI calculated from the measured velocity is also presented. To quantify the acoustic pressure from the measured velocity, the Rayleigh integral is used. As shown in the figure, the bare plate has a strongly fluctuating DI, illustrating the nonuniformly radiated sound field. Conversely, the defect $\mathrm{PnC}$ has a smooth DI close to zero within the frequency range from $2 \mathrm{kHz}$ to $5 \mathrm{kHz}$, indicating that it radiates sound uniformly.

The DI calculated from the measurements shows good agreement with the simulation at frequencies below $6 \mathrm{kHz}$. However, at frequencies above $6 \mathrm{kHz}$, deviations between the simulation and the measurement occur. The deviation may be due to the flexible motion of the studs that is not considered in the simulation.

To supplement the DI calculation in Fig. 5 the sound fields are visualized using the FE simulation. Figures 6(a)-6(c) illustrate the sound fields at three frequencies $2000 \mathrm{~Hz}, 3630 \mathrm{~Hz}$, and $4660 \mathrm{~Hz}$, where significant differences between the bare plate and the defect $\mathrm{PnC}$ are observed. For the simulation, the wave radiation condition is applied on the boundary of the acoustic domain to simulate the free space radiation. Figures 6(a)-6(c) clearly demonstrate that the defected $\mathrm{PnC}$ radiates sound more uniformly in the space.

In conclusion, we have presented an idea of using bandgap structures for the efficient and uniform radiation of sound. From numerical simulations, we found that the defect mode amplifies and smoothens the sound pressure response. This leads to increased radiation
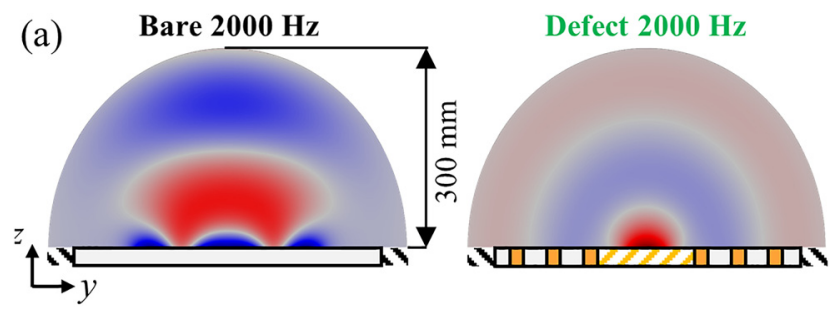

(b)
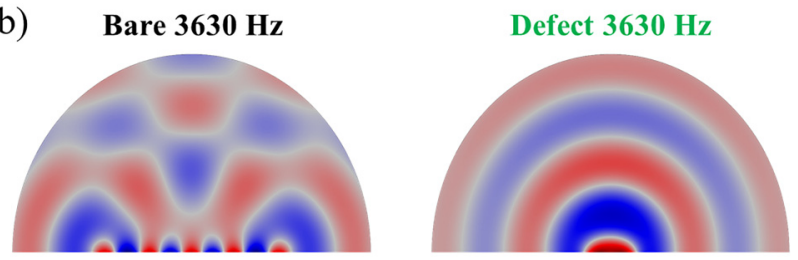

(c)

Bare $4660 \mathrm{~Hz}$

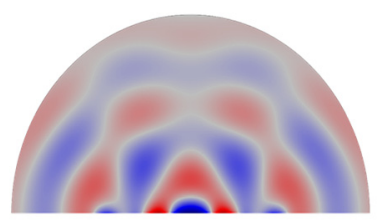

Defect $4660 \mathrm{~Hz}$

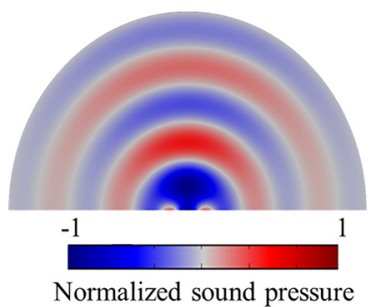

FIG. 6. The acoustic pressure fields for the bare plate and the $\mathrm{PnC}$ with defect at (a) $2000 \mathrm{~Hz}$, (b) $3630 \mathrm{~Hz}$, and (c) $4660 \mathrm{~Hz}$ 
efficiency. We presented an experimental support for the concept by measuring the vibration on the defected PnC. Sound radiation efficiency and DI are considered as performance measures. The sound radiation efficiency and the DI calculated from the measured vibration shows good agreement with the numerical simulation. We believe that this perspective will open applications of acoustic radiators. As an example of applications, a flat panel loudspeaker that plays sound using a thin panel $^{36}$ is one of the promising applications of the presented result.

This work was supported by the Technical University of Denmark (Signature project).

\section{REFERENCES}

${ }^{\mathbf{T}}$ M. Maldovan, Nature 503, 209 (2013).

${ }^{2}$ M. I. Hussein, M. J. Leamy, and M. Ruzzene, Appl. Mech. Rev. 66, 040802 (2014).

${ }^{3}$ M. M. Sigalas and E. N. Economou, J. Sound Vib. 158, 377 (1992).

${ }^{4}$ M. S. Kushwaha, P. Halevi, L. Dobrzynski, and B. Djafari-Rouhani, Phys. Rev. Lett. 71, 2022 (1993).

${ }^{5}$ M. S. Kushwaha, P. Halevi, G. Martínez, L. Dobrzynski, and B. DjafariRouhani, Phys. Rev. B 49, 2313 (1994).

${ }^{6}$ M. B. Assouar, M. Senesi, M. Oudich, M. Ruzzene, and Z. Hou, Appl. Phys. Lett. 101, 173505 (2012)

${ }^{7}$ S. Amoudache, Y. Pennec, B. Djafari-Rouhani, A. Khater, R. Lucklum, and R. Tigrine, J. Appl. Phys. 115, 134503 (2014).

${ }^{8}$ P. Celli and S. Gonella, Appl. Phys. Lett. 107(8), 081901 (2015).

${ }^{9}$ L. D’Alessandro, E. Belloni, R. Ardito, F. Braghin, and A. Corigliano, Appl. Phys. Lett. 111, 231902 (2017).

${ }^{10}$ M. Oudich, M. B. Assouar, and Z. Hou, Appl. Phys. Lett. 97, 193503 (2010).

${ }^{11}$ B. V. Damme and A. Zemp, Phys. Rev. Lett. 10, 014001 (2018).

${ }^{12}$ Z. Yang, F. Gao, X. Shi, X. Lin, Z. Gao, Y. Chong, and B. Zhang, Phys. Rev. Lett. 114, 114301 (2015).

${ }^{13}$ M. G. Baboly, A. Raza, J. Brady, C. M. Reinke, Z. C. Leseman, and I. El-Kady, Appl. Phys. Lett. 109, 183504 (2016).
${ }^{14}$ W. Jiang, D. Feng, D. Xu, B. Xiong, and Y. Wang, Appl. Phys. Lett. 109, 161102 (2016).

${ }^{15}$ J. Bonhomme, M. Oudich, B. Djafari-Rouhani, F. Sarry, Y. Pennec, B. Bonello, D. Beyssen, and P. G. Charette, Appl. Phys. Lett. 114, 013501 (2019).

${ }^{16}$ S. Zhang, L. Tin, and N. Fang, Phys. Rev. Lett. 102, 194301 (2009).

${ }^{17}$ A. Sukhovich, B. Merheb, K. Muralidharan, J. O. Vasseur, Y. Pennec, P. A. Deymier, and J. H. Page, Phys. Rev. Lett. 102, 154301 (2009).

${ }^{18}$ Y. Ruan, X. Liang, Z. Wang, T. Wang, Y. Deng, F. Qu, and J. Zhang, Appl. Phys. Lett. 114, 081908 (2019).

${ }^{19}$ X. Zhou, M. B. Assouar, and M. Oudich, Appl. Phys. Lett. 105, 233506 (2014).

${ }^{20}$ Z. Liu, X. Zhang, Y. Mao, Y. Y. Zhu, Z. Yang, C. T. Chan, and P. Sheng, Science 289(5485), 1734 (2000).

${ }^{21}$ M. Oudich, X. Zhou, and M. B. Assouar, J. Appl, Phys. 116, 193509 (2014).

${ }^{22}$ M. B. Assouar, M. Oudich, and X. Zhou, C. R. Phys. 17, 524 (2016).

${ }^{23}$ J. Jung, H.-G. Kim, S. Goo, K.-J. Chang, and S. Wang, Mech. Syst. Signal Process. 122, 206 (2019).

${ }^{24}$ L. Y. Wu, L. W. Chen, I. L. Chang, and C. C. Wang, "Acoustic energy harvesting using sonic crystals," in Advances in Energy Harvesting Methods, edited by N. Elvin and A. Erturk (Springer, New York, NY, 2013).

${ }^{25} \mathrm{M}$. Oudich and Y. Li, J. Phys. D 50, 315104 (2017).

${ }^{26}$ J. Kook and J. S. Jensen, Int. J. Solids Struct. 122, 59 (2017).

${ }^{27}$ S. Qi, M. Oudich, Y. Li, and B. Assouar, Appl. Phys. Lett. 108, 263501 (2016).

${ }^{28}$ C. Claeys, P. Sas, and W. Desmet, J. Sound Vib. 333, 14 (2014).

${ }^{29}$ H. Altug, D. Englund, and J. Vučković, Nat. Phys. 2(7), 484 (2006).

${ }^{30}$ Q. Quan, P. B. Deotare, and M. Loncar, Appl. Phys. Lett. 96(20), 203102 (2010).

${ }^{31}$ F. J. Fahy and P. Gardonio, Sound and Structural Vibration: Radiation, Transmission and Response, 2nd ed. (Academic Press, Oxford, 2007).

${ }^{32}$ S. J. Elliott and M. E. Johnson, J. Acoust. Soc. Am. 94(4), 2194 (1993).

${ }^{33}$ N. Hashimoto, Appl. Acoust. 62, 429 (2001).

${ }^{34}$ J. S. Jensen and O. Sigmund, Appl. Phys. Lett. 84, 2022 (2004).

${ }^{35}$ J. G. Tylka and E. Y. Choueiri, On the Calculation of Full and Partial Directivity Indices $3 D$ Audio and Applied Acoustics Laboratory Technical Report (Princeton University Press, Princeton, NJ, 2014).

${ }^{36}$ D. A. Anderson, M. C. Heilemann, and M. F. Bocko, J. Audio Eng. Soc. 65(9), 722-732 (2017). 